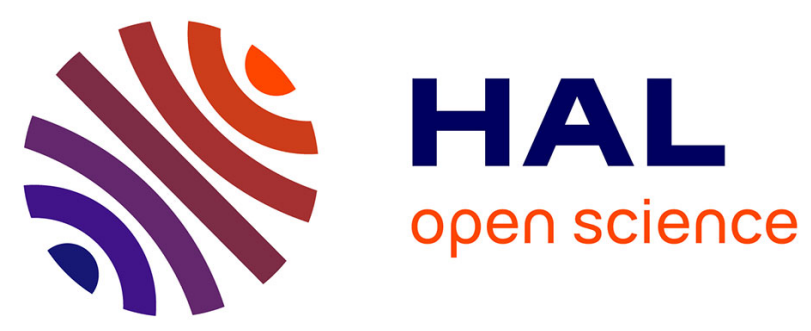

\title{
Neurally adjusted ventilatory assist as an alternative to pressure support ventilation in adults: a French multicentre randomized trial
}

\author{
A. Demoule, M. Clavel, C. Rolland-Debord, S. Perbet, N. Terzi, A. \\ Kouatchet, F. Wallet, H. Roze, F. Vargas, C. Guerin, et al.
}

\section{To cite this version:}

A. Demoule, M. Clavel, C. Rolland-Debord, S. Perbet, N. Terzi, et al.. Neurally adjusted ventilatory assist as an alternative to pressure support ventilation in adults: a French multicentre randomized trial. Intensive Care Medicine, 2016, 42 (11), pp.1723-1732. 10.1007/s00134-016-4447-8 . hal-01379329

\section{HAL Id: hal-01379329 \\ https: / hal.sorbonne-universite.fr/hal-01379329}

Submitted on 11 Oct 2016

HAL is a multi-disciplinary open access archive for the deposit and dissemination of scientific research documents, whether they are published or not. The documents may come from teaching and research institutions in France or abroad, or from public or private research centers.
L'archive ouverte pluridisciplinaire HAL, est destinée au dépôt et à la diffusion de documents scientifiques de niveau recherche, publiés ou non, émanant des établissements d'enseignement et de recherche français ou étrangers, des laboratoires publics ou privés. 


\title{
Neurally Adjusted Ventilatory Assist as an alternative to Pressure
}

\section{Support Ventilation - A multicentre randomized trial}

\author{
A Demoule ${ }^{1,2}$, M Clavel $^{3}$, C Rolland-Debord $^{1,2}$, S Perbet $^{4,5}, \mathrm{~N} \mathrm{Terzi}^{67}$, A Kouatchet $^{8}$,

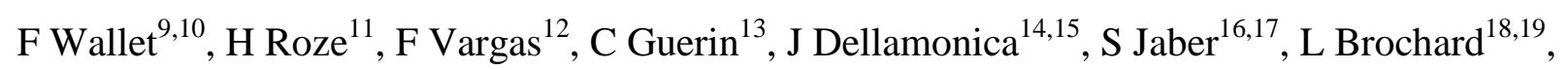
T Similowski ${ }^{1,2}$

(1) AP-HP, Groupe Hospitalier Pitié-Salpêtrière Charles Foix, Service de Pneumologie et Réanimation Médicale (Département "R3S"), F-75013, Paris, France.

(2) Sorbonne Universités, UPMC Univ Paris 06, INSERM, UMRS1158 Neurophysiologie respiratoire expérimentale et clinique, Paris, France.

(3) Réanimation polyvalente, Hôpital Dupuytren, Limoges, France.

(4) Réanimation Médico-Chirurgicale, CHU de Clermont-Ferrand, Clermont-Ferrand, France.

(5) R2D2 EA-7281, Université d'Auvergne, Clermont-Ferrand, France.

(6) INSERM U1042, Université Grenoble-Alpes, HP2, F-38000 Grenoble, France.

(7) CHU Grenoble Alpes, Service de réanimation médicale, F-38000 Grenoble, France

(8) Service de Réanimation Médicale et Médecine Hyperbare, CHU d'Angers, Angers, Angers, France.

(9) Réanimation médicale et chirurgicale, Centre hospitalier Lyon-Sud, Lyon, France.

(10) Laboratoire des Pathogènes Emergents; Centre International de Recherche en Infectiologie; Inserm U1111, CNRS UMR5308, ENS de Lyon, UCBL1

(11) Anesthésie et Réanimation, CHU de Bordeaux, Pessac, France.

(12) Réanimation médicale, Hôpital Pellegrin-Tripode, Bordeaux, France. 
(13) Réanimation médicale, Hôpital de la Croix Rousse, Lyon, France.

(14) Réanimation médicale, Hôpital de l'Archet, Centre Hospitalier Universitaire de Nice, France.

(15) INSERM 1065 team 3 C3M

(16) Anesthésie et Réanimation, Hôpital Saint-Eloi, Montpellier, France.

(17) Montpellier School of Medicine, University of Montpellier, INSERM U1046, CNRS UMR 9214, Montpellier, France.

(18) Keenan Research Centre and Li Ka Shing Institute, Saint-Michael's Hospital, Toronto, ON, Canada.

(19) Interdepartmental Division of Critical Care Medicine, University of Toronto, Canada.

\section{Authors' contributions:}

Alexandre Demoule, Thomas Similowski and Laurent Brochard designed the study. Alexandre Demoule and Thomas Similowski coordinated the study. A Demoule, M Clavel, C Rolland-Debord, S Perbet, N Terzi, A Kouatchet, F Wallet, H Roze, F Vargas, C Guerin, J Dellamonica and S Jaber, were responsible for patient screening, enrolment and follow-up. Alexandre Demoule, Laurent Brochard and Thomas Similowski wrote the manuscript. All authors had full access to all study data, contributed to drafting the manuscript or critically revised the manuscript for important intellectual content, approved the final version of the manuscript, and take responsibility for the integrity of the data and the accuracy of the data analysis.

Running title: Neurally Adjusted Ventilatory Assist in weaning 
Take home message: Neurally adjusted ventilatory assist (NAVA), a ventilatory mode that tailors the level of assistance delivered by the ventilator to the electromyographic activity of the diaphragm, can be applied efficiently in a clinical setting and reduces patient ventilator asynchrony. However, NAVA does not increase the probability of remaining in an assisted mode during the first 48 hours. Compared to pressure support ventilation, NAVA does not increase ventilator-free days and does not decrease length of stay or mortality.

Conflicts of interest: This study is an investigator-initiated trial that received financial support from Maquet. Maquet had no access to the data and was not involved in the analysis or writing of the manuscript. Alexandre Demoule has signed research contracts with Covidien, Maquet and Philips; he has also received personal fees from Covidien, Maquet and MSD. Samir Jaber has received personal fees from Dräger, Fisher and Paykel, Hamilton, and Maquet. Laurent Brochard declares research contracts with Covidien, Dräger, General Electric, as well as equipment provided by Maquet and by Philips for research protocols. Thomas Similowski belongs to the board of a research association that has received, over the past ten years, unrestricted research grants from Maquet, Hamilton, Covidien, and Philips; he is the head of a research unit (UMRS 1158) that has signed research contracts with Air Liquide Medical Systems, France; he declares no personal COI with mechanical ventilation firms; he is listed as inventor or co-inventor on several patents, granted or pending, describing a brain-ventilator interface. Marc Clavel, Camille Rolland-Debord, Sebastien Perbet, Nicolas Terzi, Achille Kouatchet, Florent Wallet, Hadrien Roze, Frederic Vargas, Claude Guerin and Jean Dellamonica have no conflict of interest related to this work. 


\begin{abstract}
Purpose. Neurally adjusted ventilatory assist (NAVA) is a ventilatory mode that tailors the level of assistance delivered by the ventilator to the electromyographic activity of the diaphragm. The objective of this study was to compare NAVA and Pressure Support Ventilation (PSV) in the early phase of weaning from mechanical ventilation.

Methods. A multicentre randomized controlled trial of 128 intubated adults recovering from acute respiratory failure was conducted in 11 intensive care units. Patients were randomly assigned to NAVA or PSV. The primary outcome was the probability of remaining in a partial ventilatory mode (either NAVA or PSV) throughout the first 48 hours without any return to assist-control ventilation. Secondary outcomes included asynchrony index, ventilator-free days and mortality.
\end{abstract}

Results. Between the NAVA and PSV groups, the proportion of patients remaining in partial ventilatory mode throughout the first 48 hours was $67.2 \% \quad$ vs. $63.3 \% \quad(\mathrm{P}=0.66)$, the asynchrony index was $14.7 \%$ vs. $26.7 \%(\mathrm{P}<0.001)$, the ventilator-free days at day 7 were 1 day [1.0-4.0] vs. 0.0 day [0.0-1.0] $(\mathrm{P}<0.01)$, the ventilator-free days at day 28 were 21 days [4-25] vs. 17 days [0-23] $(\mathrm{P}=0.12)$, the day-28 mortality rate was $15.0 \%$ vs. $22.7 \%(\mathrm{P}=0.21)$, and the rate of use of post-extubation noninvasive mechanical ventilation $43.5 \%$ vs. $66.6 \%$ $(\mathrm{P}<0.01)$.

Conclusions. NAVA is safe and feasible over a prolonged period of time but does not increase the probability of remaining in a partial ventilatory mode. However, NAVA decreases patient-ventilator asynchrony and is associated with less frequent application of post-extubation noninvasive mechanical ventilation.

Trial Registration. clinicaltrials.gov Identifier: NCT 02018666

Key words: mechanical ventilation, weaning, acute respiratory failure, neurally adjusted ventilatory assist, patient ventilator asynchrony, mortality, ventilator-free days, non invasive ventilation. 


\section{Introduction}

Partial ventilatory support is increasingly used in the intensive care unit [1]. The most widely used mode of partial ventilation is pressure support (PSV) [1], in which a constant preset level of pressure assists each inspiration regardless of the patient's inspiratory effort. Mismatch between patient respiratory drive and level of assistance can therefore occur and can be potentially harmful by causing lung overdistension and subsequent lung injury [2], respiratory discomfort [3, 4] and patient-ventilator asynchrony [5]. Neurally adjusted ventilatory assist (NAVA) delivers a pressure that is proportional to the electrical activity of the diaphragm (EAdi), a surrogate of the neural drive to breathe (see table E1 in the Online Data Supplement for a brief description of main differences between NAVA and PSV). Compared to PSV, NAVA can prevent lung overdistension [6], usually reduces patientventilator asynchrony [7] improves gas exchange [8] and restores breathing variability [9]. However, although they pledge for various substantial benefits of NAVA, all available NAVA studies were single-centre short-term studies conducted on small populations and limited to physiological outcomes. Large multicentre controlled studies with relevant clinical outcome evaluating the potential translation of these results into daily clinical practice are currently lacking. In addition, although NAVA has been successfully applied in critically ill patients for a short period of time [6-9], it is not known whether this mode could be used efficiently and safely as the main mode of partial ventilatory support.

The flexibility of NAVA and the link that it establishes between neural breathing control and ventilatory assistance could provide an advantage in terms of the patient's ability to tolerate mechanical ventilation during the early phase of partial ventilator support. The present study was therefore designed to compare NAVA and PSV during the first 48 hours following the first transition from controlled mechanical ventilation to partial ventilator support. We chose this outcome considering that weaning should be a straightforward process 
from controlled mechanical ventilation to partial ventilator support and eventually extubation and that any turn back would be a failure. The primary outcome was the likelihood for a patient to consistently remain in a partial mode during the first 48 hours without having to return to a controlled mode. Secondary outcomes were the prevalence of patient-ventilator asynchrony and major outcomes such as ventilator-free days, length of stay, and 28-day mortality.

\section{Patients and methods}

\section{Patients}

Patients receiving endotracheal mechanical ventilation for $>24 \mathrm{~h}$ for acute respiratory failure of respiratory cause (de novo hypoxaemic respiratory failure, acute cardiogenic pulmonary oedema or acute-on-chronic respiratory failure) were eligible when they met the following criteria: ability to sustain PSV for at least 30 minutes with a total level of inspiratory pressure $<30 \mathrm{cmH}_{2} \mathrm{O}$, estimated remaining duration of mechanical ventilation $>48$ $\mathrm{h}$, level of sedation $\leq 4$ on the Ramsay scale in the absence of medical decision to increase the level of sedation, fraction of inspired oxygen $\left(\mathrm{FiO}_{2}\right) \leq 50 \%$ with positive end-expiratory pressure (PEEP) $\leq 8 \mathrm{cmH}_{2} \mathrm{O}$ and absence of administration of high-dose vasopressor therapy defined by norepinephrine $>0.3 \mu \mathrm{g} \cdot \mathrm{kg}^{-1} \cdot \mathrm{min}^{-1}$ or dopamine $>10 \mu \mathrm{g} \cdot \mathrm{kg}^{-1} \cdot \mathrm{min}^{-1}$. Exclusion criteria were age younger than 18 years, known pregnancy, participation in another trial within the 30 days preceding completion of the eligibility criteria, contraindication to placement of the NAVA oesophageal tube (i.e., any contraindication to placement of a gastric tube or repositioning of a tube already in place, recent gastrointestinal suture, rupture of oesophageal varices with gastrointestinal bleeding during the 4 days prior to inclusion) and decision to withhold life-sustaining treatment. 


\section{Design}

Patients were enrolled from April 2012, to June 2013 at 11 intensive care units in France, 6 medical ICU, 4 medical surgical ICUs and one surgical ICU. The study protocol was approved for all centres by the Comité de Protection des Personnes Ile de France 6 (independent ethics committee), according to French law. Written informed consent was obtained from the patients or their surrogates before study inclusion. The trial was monitored by an independent data and safety monitoring board. Designated investigators at each centre enrolled patients. Blinding treating clinicians to group assignment was not feasible, but all analyses were conducted blindly.

As soon as patients were included, the standard nasogastric feeding tube was removed and replaced by an EAdi catheter. Patients were then randomly assigned to blocks stratified by centre to receive either PSV or NAVA. Allocation concealment was achieved using sequentially numbered, opaque, sealed envelopes.

Ventilation Strategies. The pressure support level in the PSV group and the NAVA level in the NAVA group were set to obtain a tidal volume of $6-8 \mathrm{ml} / \mathrm{kg}$ of ideal body weight. In both groups, the physician in charge set partial pressure of arterial oxygen $\left(\mathrm{FiO}_{2}\right)$ and positive end-expiratory pressure (PEEP) according to local guidelines. NAVA or PSV were continued for 14 days unless the patients met criteria for switching to controlled mechanical ventilation or for weaning and subsequent extubation. After 14 days, patients of the NAVA group were switched to PSV.

Criteria for switching to assist-control mechanical ventilation in the two groups were: 1) respiratory distress, hypoxaemia or hypercapnic acidosis despite optimization of ventilator settings; 2) severe hypotension, shock or arrhythmias; 3) increased need for sedation for agitation or for patient-ventilatory asynchrony; 4) an investigation requiring increased 
sedation; 5) Procedures that necessitated a level of sedation requiring controlled mechanical ventilation (gastrointestinal endoscopy, transoesophageal echocardiography, surgery). As soon as the criteria for switching to assist-control mechanical ventilation and the inclusion criteria were restored, partial ventilatory support with either NAVA or PSV was re-instituted according to the randomization group.

Weaning Protocol. Weaning from the ventilator was performed according to the same protocol in both groups. Although weaning could be started before the second day following randomization if deemed appropriate by the attending physician, the protocol required daily screening of prerequisite criteria for weaning at least from the second day following randomization : improvement of the acute respiratory failure causative factor, $\mathrm{SpO}_{2}>92 \%$ with $\mathrm{FiO}_{2}<50 \%$ and PEEP $<5 \mathrm{cmH}_{2} \mathrm{O}$, no infusion of vasopressor agents or sedatives and adequate responses to simple commands. If prerequisite criteria for weaning were present, a spontaneous breathing trial was performed. This test consisted of breathing spontaneously for 30 minutes to 1 hour disconnected from the ventilator on a $\mathrm{T}$ piece, or in pressure-support ventilation with an inspiratory pressure of $7 \mathrm{cmH}_{2} \mathrm{O}$ and zero end-expiratory pressure. The test was interrupted if any of the following signs of poor tolerance was observed: respiratory rate $>35 / \mathrm{min}, \mathrm{SpO}_{2}<90 \%$, heart rate $<140 / \mathrm{min}$, arterial systolic blood pressure $>180 \mathrm{mmHg}$ or $<90 \mathrm{mmHg}$. If no signs of poor tolerance were observed, the patient was extubated. Postextubation NIV could be instituted according to the decision of the physician in charge.

\section{Data Collection and Outcome Measures}

The primary outcome was the proportion of patients who remained continuously in partial ventilatory support (either NAVA or PSV) during the first 48 hours following inclusion or until extubation without any return to assist-control ventilation, which was defined as "success" (of partial ventilator support). In contrast, "failure" was defined as at least one episode of return to assist-control mechanical ventilation. To accurately assess the 
primary outcome, we precisely determined the time spent in each ventilator mode each day: data were collected each day from the ventilator with a ventilation record card, and were then further processed and stored.

Secondary outcomes were the prevalence of patient-ventilator asynchrony, the incidence of ventilator-acquired pneumonia according to predefined criteria [10], ventilatorfree days, duration of mechanical ventilation from either intubation or inclusion to successful extubation, defined as extubation not followed by another intubation within 48 hours, ICU and hospital length of stay, ICU and 28-day mortality. Application and duration of postextubation noninvasive mechanical ventilation was also collected. There were no predefined criteria for the institution post-extubation noninvasive mechanical ventilation, which was based on the decision of the physician in charge of the patient.

Data were collected at the time of randomization to characterize the severity of the underlying medical conditions, the severity of the acute illness, ventilatory settings, arterial blood gases and the cause of acute respiratory failure. Patients were monitored daily for dyspnoea as quantified by a visual analogue scale, comfort as evaluated by the ATICE score [11], ventilatory conditions and arterial blood gases.

In order to quantify patient-ventilator asynchrony, airway pressure, flow, and EAdi were acquired at $100 \mathrm{~Hz}$ for 20 minutes from the ventilator via a RS232 interface connected to a computer using commercially available software (Servo-i ${ }^{\circledR}$ RCR, version 3.6.2, Maquet Critical Care) 12, 24, 36 and 48 hours after inclusion. The five main types of asynchronies were quantified off-line by the two same investigators (AD and CRD) according to previously published definitions [7] and are described in Table E2 (see the Online Data Supplement): 1) Ineffective efforts, 2) Auto-triggering, 3) Premature cycling 4) Late cycling and 5) Double triggering. The number of episodes of each type of asynchrony was reported as the total number of each event per minute. A global asynchrony index (AI) was computed as 
previously published [5], defined as the number of asynchrony events divided by the total respiratory rate computed as the sum of the number of ventilator cycles (triggered or not) and of wasted efforts: asynchrony Index (expressed in percentage) $=$ number of asynchrony events/total respiratory rate (ventilator cycles +wasted efforts) x 100 .

\section{Statistical Analysis}

Sample size calculation was based on a previous report [12] as follows: assuming $78 \%$ of patients remaining in partial ventilator support in the PSV group during the first 48 hours, 58 patients per group would provide $80 \%$ power at a 2 -sided $\alpha$ level of 0.05 to detect a $17 \%$ absolute increase in the probability of continuously remaining on partial ventilator support in the NAVA group without any return to assist-control ventilation. With an estimated $10 \%$ failure of ventilator data collection, the final calculated sample size was 128 patients.

All analyses were conducted on an intention-to-treat basis at a 5\% level of statistical significance. Continuous data are reported as median (interquartile range [IQR]) and categorical data as number of events (percentages). Differences between groups were assessed with the $t$ test or the Mann-Whitney test for continuous variables and the $\chi^{2}$ test for categorical variables. Adjustments were performed with the Cochran-Mantel-Haenszel test. Probability of remaining continuously in an assisted mode, time-to-successful extubation defined as extubation not followed by reintubation within $48 \mathrm{~h}$, and time-to-unassisted breathing (including noninvasive ventilation) curves were constructed (Kaplan-Meier method) and differences between groups were compared using the log rank test. Patients' characteristics and baseline variables between failure and success patients were compared. All reported $p$ values are 2-sided. Statistical analysis was conducted using SAS version 9.2 statistical software (SAS Institute Inc., Cary NC, USA). 


\section{Results}

\section{Study Population}

Among 3,896 patients receiving mechanical ventilation, 128 were enrolled (median [IQR] age, 66 [57-77] years) (Figure 1, see also table E3 for volume of patients and enrolment rate per centre). Baseline characteristics of the patients are indicated in Table 1 (see also table E4 in the Online Data Supplement for an extensive description of cause of acute respiratory failure). The two study groups were balanced at baseline with regards to age, severity of illness, cause of acute respiratory failure, ventilation and oxygenation parameters. The proportion of men was higher in the NAVA group and patients of the PSV group had a higher degree of comorbidity according to the Charlson score.

Only $6 / 62$ patients $(10 \%)$ in the NAVA group and 5/66 patients (8\%) in the PSV group had successfully passed the weaning test before the second day following randomization $(P=0.71)$.

\section{Primary outcome}

For technical reasons, ventilator data collection failed in 4 patients of the NAVA group and 6 patients of the PSV group (Figure 1). Therefore, data for assessment of the primary outcome were available for 118 patients, 58 in the NAVA group and 60 in the PSV group. The proportion of patients with successful partial ventilator support who were therefore not switched at least once to assist-control ventilation during the first 48 hours following inclusion, was $67.2 \%(n=39 / 58)$ in the NAVA group vs. $63.3 \%(n=38 / 60)$ in the PSV group (relative risk $[R R]=1.19,95 \%$ confidence interval $[C I]=0.56-2.54 ; P=0.66$, Table 2 ). After adjustments based on the gender and on the Charlson score that were unbalanced between the two groups, the difference between the two groups remained non significant $(P=0.25$ and $P=0.32$, respectively). The probability of successful partial ventilator mode is shown in Figure 2 and was similar in the two groups (Log-rank test, $P=0.56$ ). The main reasons for 
switching to assist-control mechanical ventilation were similar in the two groups (see Table E5 in the Online Data Supplement). Table E6 (see Online Data Supplement) displays the patients' characteristics and baseline variables that differed significantly between failure and success patients in univariate analysis. None of these factors independently predicted the failure of partial ventilator support (either NAVA or PSV).

The time spent in each mode of mechanical ventilation during the first 48 hours following inclusion is shown on Table E7 (see Online Data Supplement). The time spent in a partial ventilator mode (NAVA or PSV) was 46.6 [43.0-48.0] $h$ in the NAVA group, including 44.1 (33.0-47.8) $\mathrm{h}$ in the NAVA mode, vs. 47.1 [39.8-48.0] $\mathrm{h}$ in the PSV group $(P=0.55)$.

\section{Respiratory Variables and dyspnoea}

Respiratory variables on days 1,2 , and 5 following randomization are reported in Table E8 (see Online Data Supplement). The initial level of assistance was a PS level of 12 (10-14) $\mathrm{cmH}_{2} \mathrm{O}$ in the PSV group and a gain of $2.0(1.0-2.5) \mathrm{cmH} 2 \mathrm{O} . \mu \mathrm{V}^{-1}$ in the NAVA group. Tidal volume, respiratory rate, minute ventilation, $\mathrm{PaO}_{2} / \mathrm{FiO}_{2}$, and $\mathrm{PaCO}_{2}$ did not differ between the two groups.

In the NAVA group, fewer patients reported dyspnoea on day $1(P=0.03)$. ATICE comfort score did not differ between groups.

\section{Patient-ventilator asynchrony}

For technical reasons, flow, pressure and EAdi recording failed in 9 patients of the NAVA group and 16 patients of the PSV group. Data concerning the prevalence of the main asynchronies were therefore available for 103 patients, 53 in the NAVA group and 50 in the PSV group. Figure 3 shows the prevalence of the main patient-ventilator asynchronies observed in the two groups. The asynchrony index was significantly lower in the NAVA group than in the PSV group $(26.7[15.8-45.1] \%$ vs. $14.7[12.3-21.7] \%, P<0.001)$. Ineffective 
efforts and late cycling were more frequent in the PSV group, whereas double triggering was more frequent in the NAVA group.

\section{Other secondary outcomes}

The spontaneous breathing trial was performed pressure-support ventilation in 115 patients and disconnected from the ventilator on a T piece in 13 patients. Differences between groups were not significant for ICU mortality (RR for NAVA, 0.61 [95\% CI, 0.27-1.35]), hospital mortality (RR, 0.68 [95\% CI, 0.32-1.47]), 28-day mortality (RR 0.64[95\% CI, 0.301.35]), ICU length of stay and hospital length of stay, and the relative risk of acquiring ventilator-acquired pneumonia was 2.66 [95\% CI, 0.54-13.22]) (Table 2). The number of ventilator-free days was lower in the NAVA group on day 7 , but not on day 14 or day 28 . The probability of successful extubation and the probability of breathing without assistance are shown in Figure 4 and were similar in the two groups (Log-rank test, $\mathrm{p}=0.49$ ). In the NAVA group compared to the PSV group, fewer patients received post-extubation noninvasive mechanical ventilation (RR, 0.65 [95\% CI, 0.47-0.91]) and for a shorter duration (Table 2).

\section{Adverse events}

Four adverse events possibly related to the NAVA catheter were recorded: one in the NAVA group (one pneumothorax) and three in the PSV group (one pneumothorax, one gastrointestinal hemorrhage and one laryngeal edema). The pneumothorax occurring in the PSV group was directly related to a misplacement of the NAVA tube. No adverse events related to the NAVA mode were recorded.

\section{Discussion}

The main findings of our study can be summarized as follows: 1) NAVA did not increase the probability of remaining in a partial ventilator mode compared to PSV; 2) NAVA improved patient-ventilator synchrony and was associated with less dyspnoea and less 
frequent application for post-extubation NIV; 3) NAVA did not reduce the duration of mechanical ventilation or ICU mortality; 4) NAVA can be delivered safely and efficiently for several days.

The NAVA mode, first described more than 15 years ago [13], has been available for clinical use for almost 7 years. To our knowledge, the present study is the first to describe the use of NAVA in a large cohort of patients recovering from acute respiratory failure. Although many studies have previously investigated NAVA in ICU patients, they were conducted in small samples of patients, who were rarely studied for more than a few hours [14]. In the present study, NAVA was extensively described during the first 48 hours, and was continued for 14 days unless the patients met criteria for switching to controlled mechanical ventilation or for weaning and subsequent extubation. One serious adverse event was observed, a pneumothorax caused by the misplacement of the NAVA catheter in the PSV group. Our data show that NAVA can be applied safely and efficiently in routine care instead of PSV. It is noteworthy that patients in the NAVA group mostly remained on NAVA with few switches to either PSV or assist-control ventilation. However, NAVA did not improve the probability of remaining in an assisted mode of breathing compared to PSV and the reasons for switching back to a controlled mode were similar in the two groups. In addition, the total time spent in a controlled mode was similar in the two groups. Why NAVA failed to improve the probability and the time spent in an assisted mode remains unclear. A number of hypotheses can be proposed. First, various precipitating factors of acute respiratory failure were observed. It is possible that NAVA is mostly beneficial in specific patient groups, such as COPD, patients with severe patient-ventilator asynchrony or patients who are difficult-to-wean. Second, all participating centres were experienced with mechanical ventilation. Therefore, the proportion of patients in the PSV arm who did not remain constantly in PSV could have been sufficiently low to prevent any additional improvement. After removal of failures of partial ventilator 
mode (either NAVA or PSV) due to sedation for the purposes of an investigation, comparison with previous reports from experienced teams showed a similar partial ventilator mode failure rate $[12,15]$. Finally, NAVA failed to reduce the duration of mechanical ventilation and ICU mortality, but our study was not powered to detect such differences.

NAVA improved patient-ventilator synchrony over the first $48 \mathrm{~h}$. Patient-ventilator asynchrony is potentially an important outcome, as a high prevalence of asynchrony is associated with longer duration of mechanical ventilation and a higher mortality rate $[5,16$, 17]. The benefit of NAVA on patient-ventilator interactions has been previously described in small single-centre physiology studies $[7,18]$. Our results tend to extend these findings by the multicentre design of this study. We also found a lower prevalence of dyspnoea in the NAVA group on the first day following randomization, but not thereafter, which could be related to improved matching between respiratory neural drive and assistance during a transition period of maximal lability. Finally, we found that fewer patients in the NAVA group received postextubation noninvasive mechanical ventilation and for a shorter duration. Consequently, the number of ventilator-free days on day 7 was lower in the NAVA group. The relevance of this noticeable finding needs to be confirmed by further studies since there were no predefined criteria for the institution post-extubation noninvasive mechanical ventilation, which was based on the decision of the physician in charge of the patient.

The strengths of this trial include a rigorous method to avoid bias such as the recording of the ventilator trends with a card that allows precise calculation of the time spent on each mode and predefined criteria for initiation of a spontaneous breathing trial and for extubation. The participation of 11 ICUs enhances the generalisability of our findings. Finally, this is the first study to demonstrate the feasibility and safety of NAVA for several days rather than for just a few hours.

Limitations of the trial include the recommended NAVA level setting that targeted a 
range of tidal volume. The relevance of this setting has been questioned and alternative rules have been proposed $[14,19]$. However, these rules are still quite complex to use and timeconsuming to apply. Targeting a tidal volume remains an easy way to set NAVA and is easily compatible with routine clinical practice. In addition, one could argue that our study was underpowered. However, the difference between the two groups was small and a very high sample size would be required to detect such a difference. We feel that our primary outcome may not be the best one to describe a difference between the two groups. The fact that ventilator asynchrony was calculated based on four recordings performed on the two days following inclusion is another limitation. Indeed, a recent work shows that asynchrony may occur at anytime and that should be quantified over the whole duration of mechanical ventilation [16]. For technical reasons (failure of recordings), patient ventilator asynchrony could not be detected in 25 patients. These missing data could alter the veracity of our results. To solve this issue, we perform simulation. Markov chain Monte Carlo was first preformed to produce monotone missingness followed by multiple imputations with the predictive mean matching method (for Asynchrony Index, $P=0.062$ ). Finally, although postoperative patients were included, they all had a complication of surgery involving the respiratory system or prolonged mechanical ventilation.

In summary, in patients recovering from acute respiratory failure, we found that NAVA was safe and feasible over a prolonged period of time, but we failed to find a difference in the efficacy of NAVA and PSV to maintain patients in a partial ventilatory mode. We confirmed that NAVA reduces the prevalence of patient-ventilator asynchrony, and observed that it does so in a sustained manner. Our findings of improved dyspnoea on day-1 under NAVA and decreased use of post-extubation noninvasive mechanical ventilation after NAVA provide guidance for the design of future trials. 


\section{References}

1. Esteban A, Frutos-Vivar F, Muriel A, Ferguson ND, Penuelas O, Abraira V, Raymondos K, Rios F, Nin N, Apezteguia C, Violi DA, Thille AW, Brochard L, Gonzalez M, Villagomez AJ, Hurtado J, Davies AR, Du B, Maggiore SM, Pelosi P, Soto L, Tomicic V, D'Empaire G, Matamis D, Abroug F, Moreno RP, Soares MA, Arabi Y, Sandi F, Jibaja M, Amin P, Koh Y, Kuiper MA, Bulow HH, Zeggwagh AA, Anzueto A (2013) Evolution of mortality over time in patients receiving mechanical ventilation. Am J Respir Crit Care Med 188: 220-230

2. $\quad$ Slutsky AS, Ranieri VM (2013) Ventilator-induced lung injury. N Engl J Med 369: 2126-2136

3. Schmidt M, Banzett RB, Raux M, Morelot-Panzini C, Dangers L, Similowski T, Demoule A (2014) Unrecognized suffering in the ICU: addressing dyspnea in mechanically ventilated patients. Intensive Care Med 40: 1-10

4. Schmidt M, Kindler F, Gottfried SB, Raux M, Hug F, Similowski T, Demoule A (2013) Dyspnea and surface inspiratory electromyograms in mechanically ventilated patients. Intensive Care Med 39: 1368-1376

5. Thille AW, Rodriguez P, Cabello B, Lellouche F, Brochard L (2006) Patientventilator asynchrony during assisted mechanical ventilation. Intensive Care Med 32: $1515-1522$

6. Schmidt M, Kindler F, Cecchini J, Poitou T, Morawiec E, Persichini R, Similowski T, Demoule A (2015) Neurally adjusted ventilatory assist and proportional assist ventilation both improve patient-ventilator interaction. Crit Care 19: 56

7. Piquilloud L, Vignaux L, Bialais E, Roeseler J, Sottiaux T, Laterre PF, Jolliet P, Tassaux D (2010) Neurally adjusted ventilatory assist improves patient-ventilator interaction. Intensive Care Med 
8. Coisel Y, Chanques G, Jung B, Constantin JM, Capdevila X, Matecki S, Grasso S, Jaber S (2010) Neurally adjusted ventilatory assist in critically ill postoperative patients: a crossover randomized study. Anesthesiology 113: 925-935

9. Schmidt M, Demoule A, Cracco C, Gharbi A, Fiamma MN, Straus C, Duguet A, Gottfried SB, Similowski T (2010) Neurally adjusted ventilatory assist increases respiratory variability and complexity in acute respiratory failure. Anesthesiology 112: $670-681$

10. Society AT (2005) Guidelines for the management of adults with hospital-acquired, ventilator-associated, and healthcare-associated pneumonia. Am J Respir Crit Care Med 171: 388-416

11. De Jonghe B, Cook D, Griffith L, Appere-de-Vecchi C, Guyatt G, Theron V, Vagnerre A, Outin H (2003) Adaptation to the Intensive Care Environment (ATICE): Development and validation of a new sedation assessment instrument. Crit Care Med 31: $2344-2354$

12. Xirouchaki N, Kondili E, Vaporidi K, Xirouchakis G, Klimathianaki M, Gavriilidis G, Alexandopoulou E, Plataki M, Alexopoulou C, Georgopoulos D (2008) Proportional assist ventilation with load-adjustable gain factors in critically ill patients: comparison with pressure support. Intensive Care Med 34: 2026-2034

13. Sinderby C, Navalesi P, Beck J, Skrobik Y, Comtois N, Friberg S, Gottfried SB, Lindstrom L (1999) Neural control of mechanical ventilation in respiratory failure. Nat Med 5: 1433-1436

14. Tuchscherer D, Z'Graggen W J, Passath C, Takala J, Sinderby C, Brander L (2011) Neurally adjusted ventilatory assist in patients with critical illness-associated polyneuromyopathy. Intensive Care Med 37: 1951-1961 
15. Cereda M, Foti G, Marcora B, Gili M, Giacomini M, Sparacino ME, Pesenti A (2000) Pressure support ventilation in patients with acute lung injury. Crit Care Med 28: $1269-1275$

16. Blanch L, Villagra A, Sales B, Montanya J, Lucangelo U, Lujan M, Garcia-Esquirol O, Chacon E, Estruga A, Oliva JC, Hernandez-Abadia A, Albaiceta GM, FernandezMondejar E, Fernandez R, Lopez-Aguilar J, Villar J, Murias G, Kacmarek RM (2015) Asynchronies during mechanical ventilation are associated with mortality. Intensive Care Med 41: 633-641

17. de Wit M, Miller KB, Green DA, Ostman HE, Gennings C, Epstein SK (2009) Ineffective triggering predicts increased duration of mechanical ventilation. Crit Care Med 37: 2740-2745

18. Spahija J, de Marchie M, Albert M, Bellemare P, Delisle S, Beck J, Sinderby C (2010) Patient-ventilator interaction during pressure support ventilation and neurally adjusted ventilatory assist. Crit Care Med 38: 518-526

19. Brander L, Leong-Poi H, Beck J, Brunet F, Hutchison SJ, Slutsky AS, Sinderby C (2009) Titration and implementation of neurally adjusted ventilatory assist in critically ill patients. Chest 135: 695-703 


\section{Figure legends}

Figure 1. Flow diagram of the trial

EAdi, electromyographic activity of the diaphragm; NIV, noninvasive ventilation.

Figure 2. Kaplan-Meier estimates of probability of continuously remaining in partial ventilatory support (either NAVA or PSV) without any return to assist-control ventilation during the first $\mathbf{4 8}$ hours following randomization

NAVA, neurally adjusted ventilatory assist; PSV, pressure support ventilation.

Figure 3. Rates and prevalence of main patient ventilator asynchronies

On the left panel, box plots showing the median and interquartile range for the prevalence of the main patient ventilator asynchronies. On the right panel, box plots showing the median and interquartile range for the asynchrony index (AI).

$* \mathrm{p}<0.05$

Figure 4. Kaplan-Meier estimates of probability of successful extubation (left panel) and probability of breathing without assistance (right panel) from the day of randomization to day 28

Successful extubation was defined as extubation not followed by reintubation within $48 \mathrm{~h}$.

NAVA, neurally adjusted ventilatory assist; PSV, pressure support ventilation. 
5883 admissions

3896 received mechanical ventilation assessed for eligibility

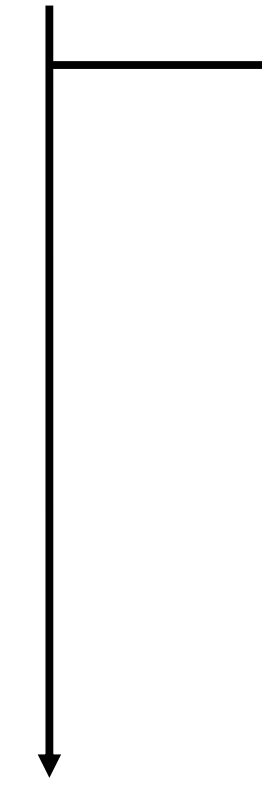

3550 did not meet entry criteria

- 628 received only NIV

- 1923 received invasive mechanical ventilation for a non-respiratory cause

- 703 estimated remaining mechanical ventilation $<48 \mathrm{hrs}$

- 296 died, tracheostomized, or extubated before meeting inclusion criteria

127 met $\geq 1$ other exclusion criteria

- 41 contra indication to NAVA catheter

- 29 recent gastrointestinal suture

- 12 rupture of esophageal varices

- $86 \geq 1$ other exclusion criteria

91 not included for technical reasons

62 randomized to

receive NAVA

62 received NAVA

62 were analyzed

Ventilator trends data collection failed in 4 patients Primary outcome could be analyzed in 58 patient

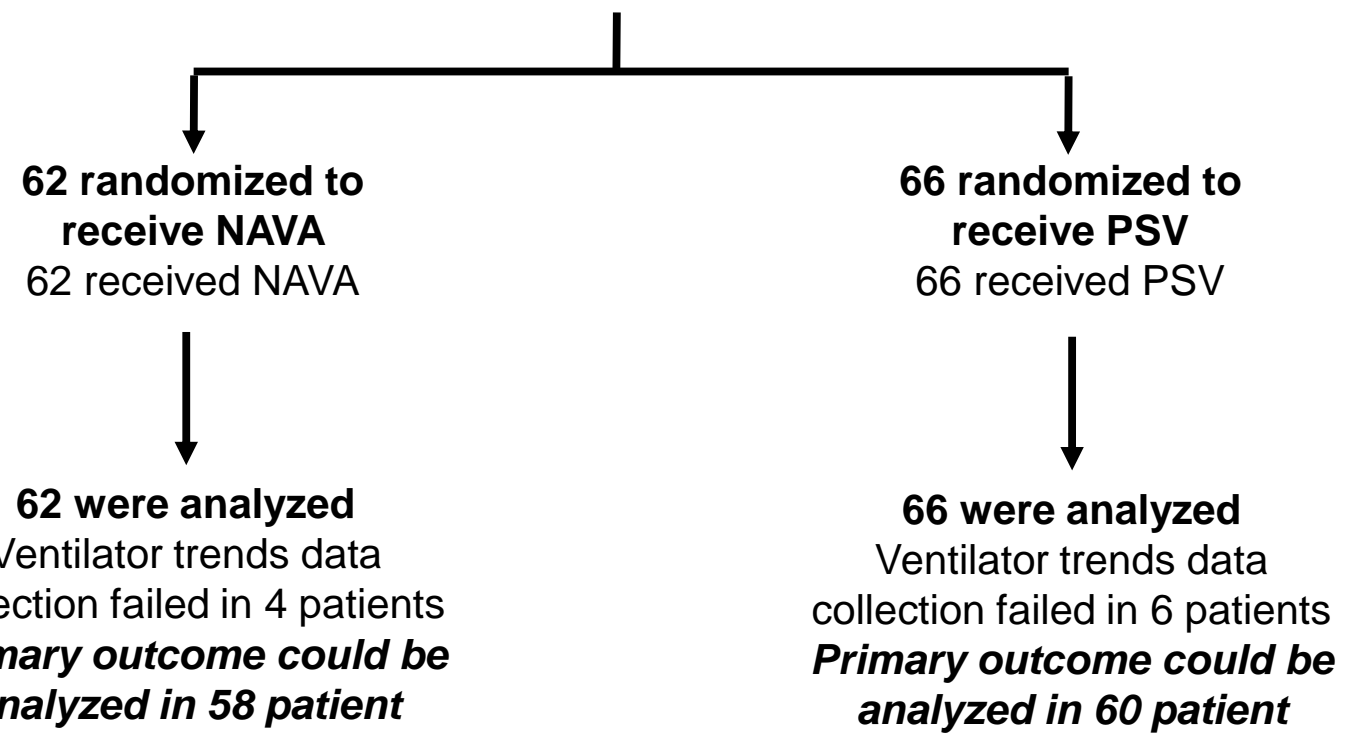

128 randomized

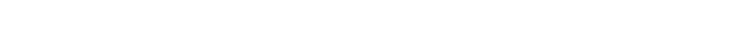


Continuously remaining in partial ventilatory support

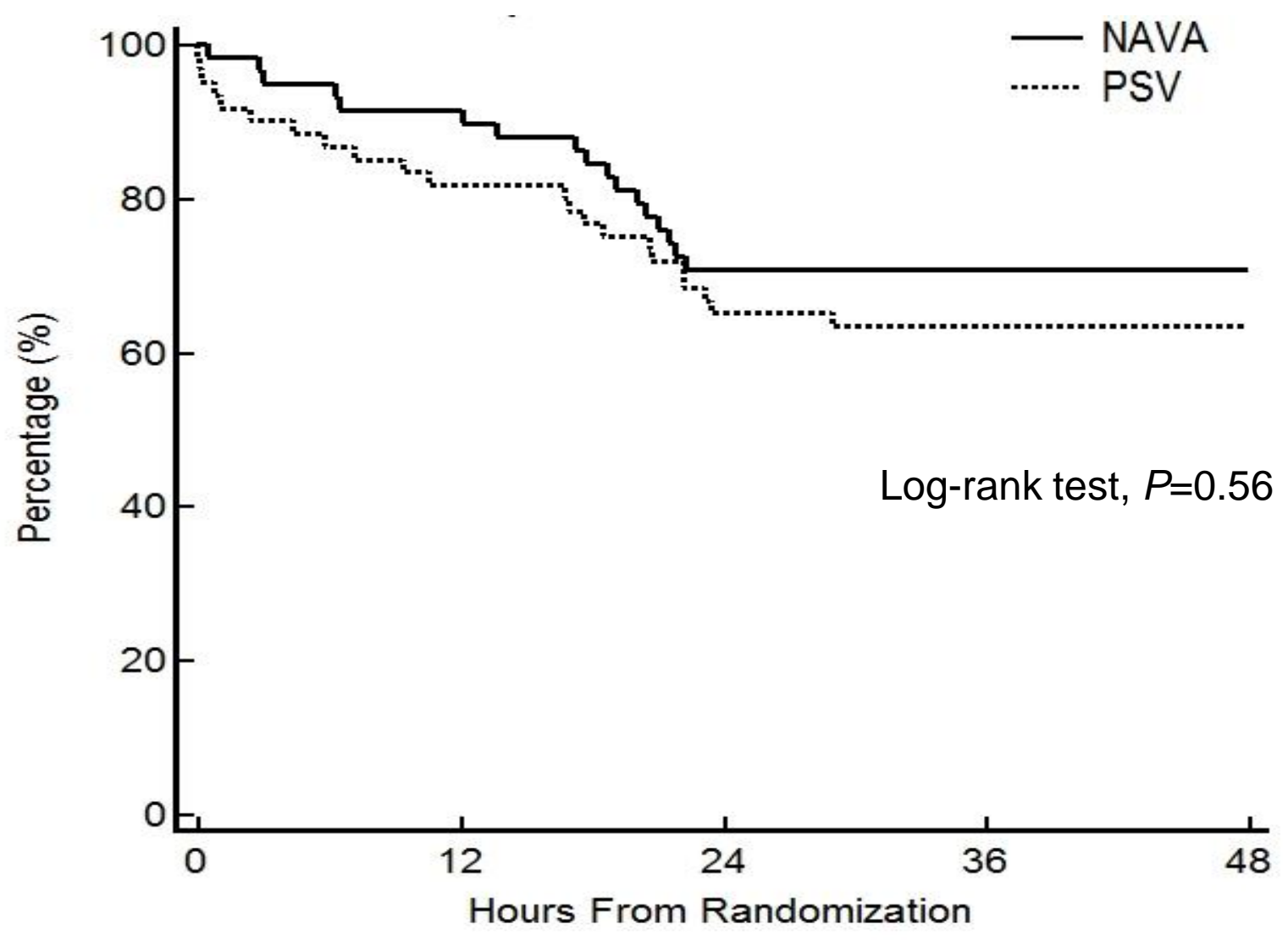




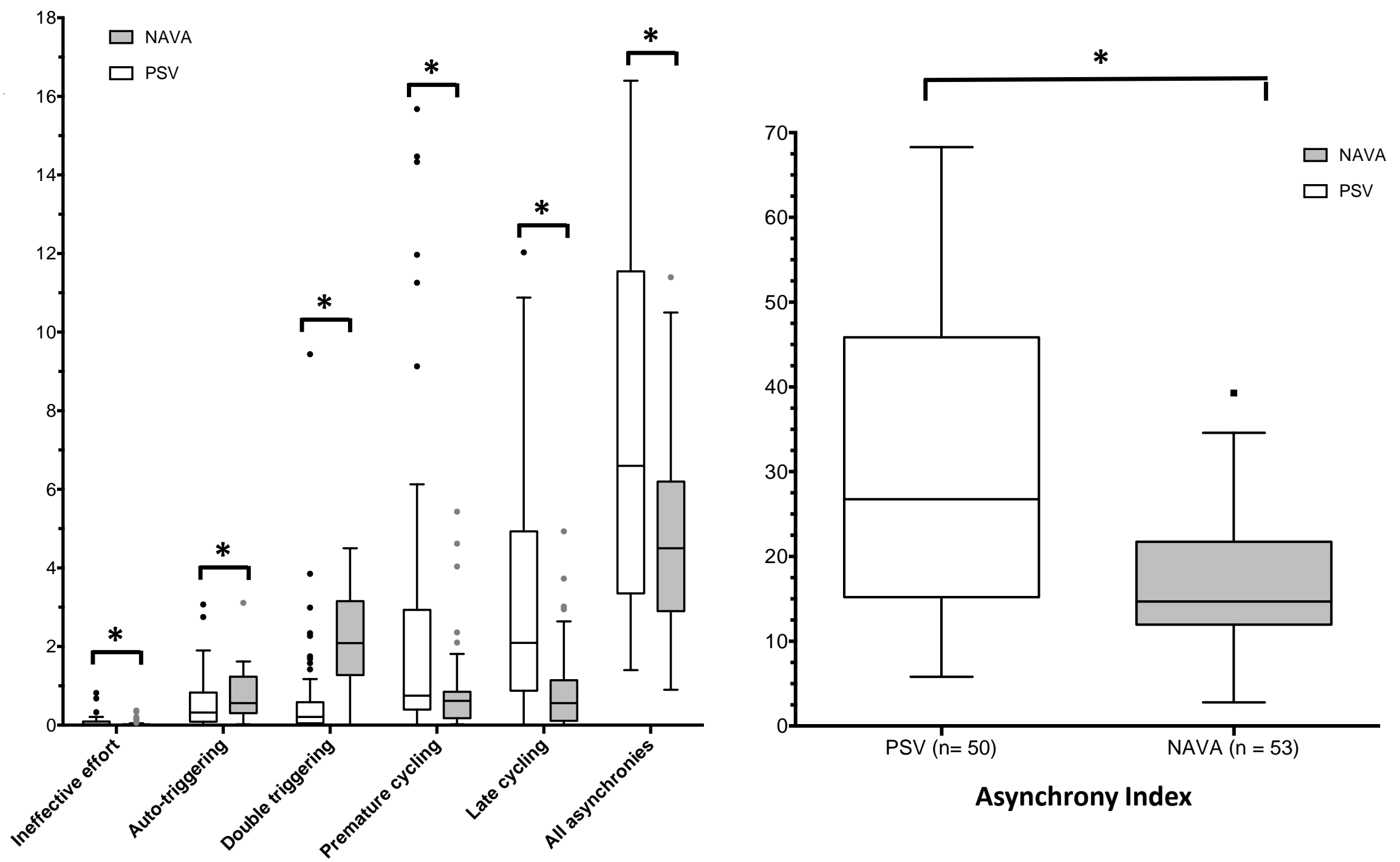



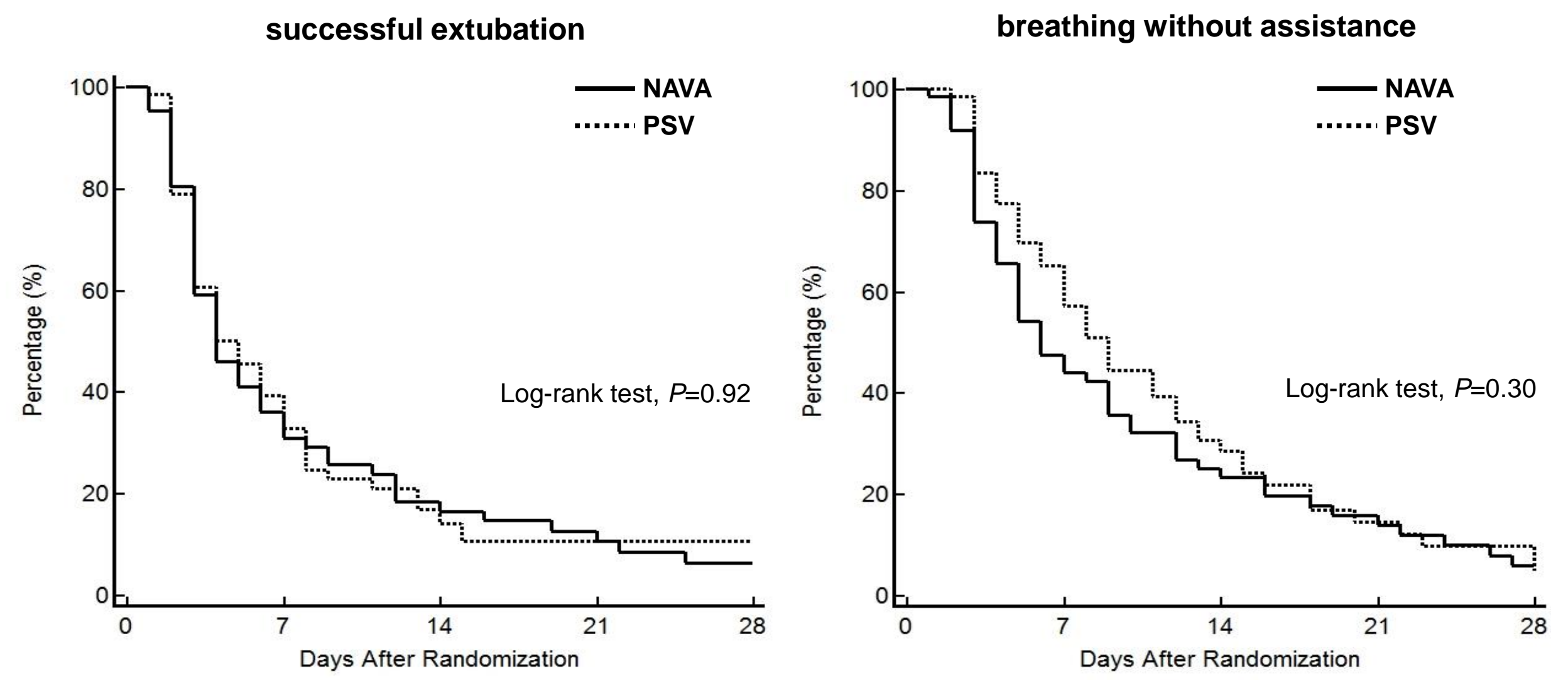


\section{Tables}

Table 1. Baseline characteristics of the patients

\begin{tabular}{|c|c|c|c|}
\hline & $\begin{array}{c}\text { PSV } \\
(n=66)\end{array}$ & $\begin{array}{l}\text { NAVA } \\
(n=62)\end{array}$ & $\mathbf{P}$ \\
\hline Gender, $M$ & $39(59)$ & $47(76)$ & 0.04 \\
\hline Age, years & $64(53-77)$ & $66(61-77)$ & 0.30 \\
\hline SAPS 2 & $43.5(34-59)$ & $44(35-60)$ & 1.00 \\
\hline Charlson score & $5.5(5-8)$ & $5(4-5)$ & 0.04 \\
\hline $\begin{array}{l}\text { Duration of mechanical ventilation } \\
\text { prior to inclusion, days }\end{array}$ & $5(3-8)$ & $4(2-8)$ & 0.21 \\
\hline Cause of acute respiratory failure & & & 0.75 \\
\hline De novo, $n(\%)$ & $38(58)$ & $34(55)$ & \\
\hline Postoperative, $n(\%)$ & $13(20)$ & $13(21)$ & \\
\hline Acute-on-chronic, $n(\%)$ & $12(18)$ & $12(19)$ & \\
\hline $\begin{array}{l}\text { Acute cardiogenic } \\
\text { pulmonary oedema, } n(\%)\end{array}$ & $3(5)$ & $3(5)$ & \\
\hline \multicolumn{4}{|l|}{ Ventilator settings } \\
\hline PEEP, $\mathrm{CmH}_{2} \mathrm{O}$ & $6(5-8)$ & $6(5-8)$ & 0.16 \\
\hline Pressure support level, $\mathrm{cmH}_{2} \mathrm{O}$ & $12(10-14)$ & $12(10-16)$ & 0.84 \\
\hline \multicolumn{4}{|l|}{ Respiratory measures } \\
\hline Respiratory rate $^{\mathrm{a}}, \mathrm{min}^{-1}$ & $24(20-27)$ & $24(19-29)$ & 0.71 \\
\hline Tidal volume, $m l$ & $450(380-511)$ & $450(407-421)$ & 0.29 \\
\hline Tidal volume, $\mathrm{ml} . \mathrm{kg}^{-I}$ & $7.0(6.0-8.2)$ & $7.1(6.5-8.2)$ & 0.33 \\
\hline Minute ventilation, $l . \min ^{-I}$ & $10(8.9-12)$ & $11(8.6-14)$ & 0.38 \\
\hline \multicolumn{4}{|l|}{ Blood gases } \\
\hline $\mathrm{PaO}_{2} / \mathrm{FiO}_{2}, \mathrm{mmHg}$ & $227(192-286)$ & $235(185-265)$ & 0.87 \\
\hline pH & $7.43(7.39-7.46)$ & $7.43(7.39-7.46)$ & 0.64 \\
\hline $\mathrm{PaCO}_{2}, \mathrm{mmHg}$ & $40(35-45)$ & $39(34-47)$ & 0.74 \\
\hline
\end{tabular}

PSV, pressure support ventilation; NAVA, neurally adjusted ventilatory assist; ARF, acute respiratory failure; SAPS, simplified acute physiology score; ICU, intensive care unit; PEEP, positive end-expiratory pressure.

${ }^{a}$ Respiratory rate was computed based on ventilator breaths.

Continuous data are reported as median (interquartile range $[\mathrm{IQR}]$ ) and categorical data are reported as number of events (percentages). 
Table 2. Primary and secondary endpoints

\begin{tabular}{|c|c|c|c|}
\hline & $\begin{array}{c}\text { PSV } \\
(n=66)\end{array}$ & $\begin{array}{l}\text { NAVA } \\
(\mathrm{n}=62)\end{array}$ & $\mathbf{P}$ \\
\hline $\begin{array}{l}\text { Proportion of patients with successful } \\
\text { partial ventilator support } \mathrm{t}^{\mathrm{a}}, n(\%)\end{array}$ & $38 / 60(63.3)$ & $39 / 58(67.2)$ & 0.66 \\
\hline $\begin{array}{l}\text { Duration of invasive mechanical } \\
\text { ventilation, days }\end{array}$ & $10.0(7.0-16.0)$ & $10.0(6.0-17.0)$ & 0.58 \\
\hline Duration of mechanical ventilation*, days & $13.5(10.0-21.0)$ & $12.0(7.0-17.0)$ & 0.12 \\
\hline $\begin{array}{l}\text { Days of invasive mechanical ventilation } \\
\text { from randomization }\end{array}$ & $4.0(3.0-8.0)$ & $4.0(3.0-9.0)$ & 0.19 \\
\hline $\begin{array}{l}\text { Days of mechanical ventilation from } \\
\text { randomization }^{\mathrm{b}}\end{array}$ & $8.05 .0-13.0)$ & $6.0(3.0-12.5)$ & 0.19 \\
\hline Invasive ventilator-free days, day 7 & $3.0(0.0-4.0)$ & $3.0(0.0-4.0)$ & 0.87 \\
\hline Ventilator-free days $^{\mathrm{b}}$, day 7 & $0.0(0.0-1.0)$ & $1.0(0.0-4.0)$ & 0.01 \\
\hline Invasive ventilator-free days, day 14 & $9.0(4.8-11.0)$ & $10.0(2.5-11.0)$ & 0.91 \\
\hline Ventilator-free days $^{\mathrm{b}}$, day 14 & $5.0(0.0-9.3)$ & $8.0(1.0-11.0)$ & 0.09 \\
\hline Invasive ventilator-free days, day 28 & $23.0(13.8-25.0)$ & $24.0(10.5-25.0)$ & 0.76 \\
\hline Ventilator-free days $^{\mathrm{b}}$, day 28 & $18.0(0.5-23.0)$ & $21.0(6.5-25.0)$ & 0.09 \\
\hline Ventilator-acquired pneumonia, $n(\%)$ & $2(3.0 \%)$ & $5(8.3 \%)$ & 0.26 \\
\hline Days of ICU stay & $18.0(12.0-26.0)$ & $18.0(13.5-32.0)$ & 0.48 \\
\hline Days of hospital stay & $31.0(23.0-37.0)$ & $34.0(23.0-40.0)$ & 0.58 \\
\hline Death before ICU discharge, $n(\%)$ & $14(21.2)$ & $8(12.9)$ & 0.21 \\
\hline Death in the first 28 days, $n(\%)$ & $15(22.7)$ & $9(14.5)$ & 0.25 \\
\hline Use of post-extubation NIV, $n(\%)$ & $44(66.6)$ & $27(43.5)$ & 0.008 \\
\hline Days of post-extubation NIV & $2.0(0.0-4.0)$ & $0.0(0.0-3.0)$ & 0.03 \\
\hline
\end{tabular}

PSV, pressure support ventilation; NAVA, neurally adjusted ventilator assist; NIV, noninvasive ventilation; ICU, intensive care unit.

${ }^{a}$ Proportion of patients with successful partial ventilator support who were therefore not switched at least once to assist-control ventilation during the first 48 hours following inclusion 
${ }^{\mathrm{b}}$ Including noninvasive ventilation

Continuous data are reported as median (interquartile range [IQR]) and categorical data are reported as number of events (percentages). 